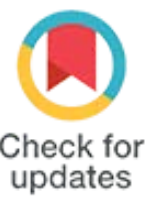

DOI: $10.29252 / \mathrm{ijcp}-25695$

\section{Idiopathic Chylopericardium: A Case Report and Brief Review of Literature}

\author{
Majda Haraka ${ }^{1}{ }^{*}$, Monia El Mourid ${ }^{1}$, Amine Mamoun Boutaleb ${ }^{1}$, \\ Khadija Khay ${ }^{1}$, Leila Azzouzi ${ }^{1}$, Rachida Habbal ${ }^{1}$ \\ ${ }^{1}$ Ibn Roch University Hospital, Casablanca, Morocco \\ * Corresponding author: Majda Haraka, Ibn Roch University Hospital, \\ Casablanca, Morocco. E-mail: majdaharaka5204@gmail.com
}

\begin{tabular}{|c|c|}
\hline Submitted: $29-05-2019$ & \multirow{7}{*}{$\begin{array}{l}\text { Abstract } \\
\text { Chylopericardium is the accumulation of chylous fluid in the pericardial space. Many } \\
\text { etiologies can be found and the treatment depends on the etiology and remains unclear. } \\
\text { This rare entity needs some tests to determine its causes. Diagnosis is based on many } \\
\text { tools; the pericardial biopsy puncture and lymphoscintigraphy are the most important } \\
\text { ones. The treatment requires low fat diet, lipid lowering therapy and surgery in some } \\
\text { cases. We here report the first case in the IBN ROCHD university hospital's Cardiology } \\
\text { department of Casablanca, Morocco. It deals with a } 32 \text {-year-old patient who consulted } \\
\text { for dyspnea exacerbation for } 18 \text { months, becoming at rest. Chest X-ray and } \\
\text { echocardiography were performed discovering pericardial effusion. Pericardiocentesis } \\
\text { was performed to confirm the nature of liquid and lymphoscintigraphy showed no } \\
\text { abnormal communication between the thoracic duct and pericardium. }\end{array}$} \\
\hline Accepted: 20-06-2019 & \\
\hline Keywords: & \\
\hline Pericardial Effusion & \\
\hline Chylopericardium & \\
\hline Pericardium & \\
\hline $\begin{array}{l}\text { (C) 2019. International Journal } \\
\text { of Cardiovascular Practice. }\end{array}$ & \\
\hline
\end{tabular}

\title{
INTRODUCTION
}

Chylopericardium (CP) is accumulation of chylous fluid in the pericardial cavity [1]. It is considered idiopathic or primary when no etiology is found after investigations. The treatment often requires lipidlowering therapy and surgical drainage of the effusion. We report a 32-year-old man admitted in the IBN ROCHD University Hospital's cardiology department of Casablanca, for idiopathic chylopericardium.

\section{CASE REPORT}

A 32-year-old man was admitted with no cardiovascular risk factor of pericardial effusion, no sign of systemic disease, or past history of tuberculosis except for a thoracic trauma 3 years before with no complications. He complained dyspnea of NYHA class II, evolving for 18 months and decreasing at an anteflexed position. The worsening symptoms during the two last months led patient to seek medical consultation. Chest $\mathrm{X}$ - ray was performed showing cardiomegaly and echocardiography revealed liquid pericardial effusion of $16 \mathrm{~mm}$ in front of the right ventricle, $18 \mathrm{~mm}$ in front of the right atrium and $28 \mathrm{~mm}$ in front of the left ventricle without any sign of compression. His blood pressure was $120 / 80 \mathrm{mmHg}$ and heart rate $95 \mathrm{Bpm}$. There was no jugular vein distension or other signs of tamponade.
ECG showed a sinus rhythm, with a low voltage and electrical alternans.

A computed tomography scan was performed showing pericardial effusion with no other abnormalities (Fig 1).

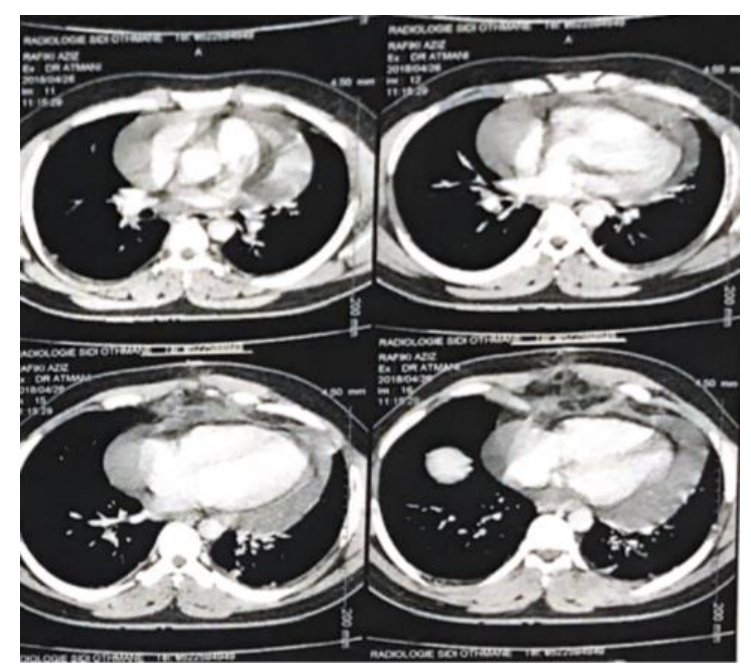

Figure 1: Axial Tomodensitometric Section passing through the anterior Mediastinum objectifying a Pericardial Effusion of Average Abundance

Blood count, electrolytes, kidney function, and liver function tests had normal findings. Immunological 
assessment, thyroid function tests and tuberculosis had negative results. Lipid profile demonstrated cholesterol of $5.4 \mathrm{mmol} / \mathrm{l}$, triglycerides of $1.9 \mathrm{mmol} / \mathrm{l}$, low density lipoprotein (LDL)-cholesterol of $2.8 \mathrm{mmol} / 1$ and highdensity lipoprotein (HDL) -cholesterol of $1.4 \mathrm{mmol} / \mathrm{l}$. A pericardial biopsy puncture was performed and 800 $\mathrm{mL}$ of thick milky chylous fluid was drained. The chylous nature of the fluid was confirmed by high level of triglycerides $(10 \mathrm{mmol} / \mathrm{L})$ and a cholesteroltriglyceride ratio of 0.3 (below 1). Cytology demonstrated abundance of lymphocytes and no evidence of malignant cells. The culture for bacteria had negative result and anatomopathological study showed a chronic fibrous pericarditis with no sign of malignancy. A lymphoscintigraphy was performed, on a GE 630 Gamma Camera, to look for a breach in the thoracic duct or a communication with the pericardium. The protocol was a subcutaneous injection at the first interdigital space of each foot and each hand at rest of $90 \mathrm{MBq}$ Nancoll labeled with Tc 99m. It confirmed a normal lymphatic drainage in the upper and lower limbs with the absence of scintigraphic argument that may explain chylous pericardial effusion (Fig 2).

The patient received lipid lowering treatment and antiinflammatory dosage of aspirin, colchicine $1 \mathrm{mg}$ for 6 months and assigned to low fat diet with no improvement. Therefore, an anti-bacillary treatment was started for 6 months, since the patient was in an endemic country for tuberculosis. 3 months later, the patient came back with recurrence of the pericardial effusion without any sign of compression or tamponade. The inferior vena cava measured $21 \mathrm{~mm}$. Therefore, the patient was candidate for a pleuro-pericardial window with a favorable evolution for 3 months till writing the manuscript.
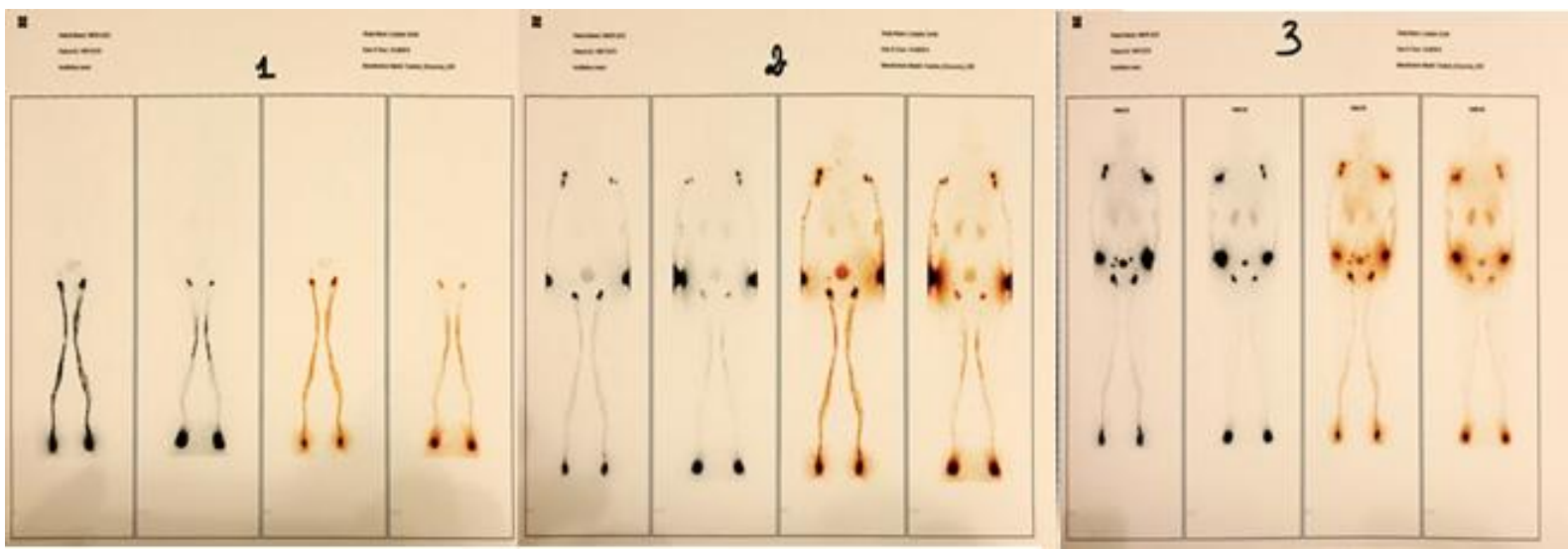

Figure 2: Lymphoscintigraphic Images showing Normal Lymphatic Drainage

\section{DISCUSSION}

Chylopericardium is an abnormal accumulation of chylous fluid containing triglycerides in high concentrations in the pericardium [2]. It is still a rare form of pericardial effusion. Only few cases are described in literature; the first one was by Hasebrock in 1888 [3] and then it was reviewed in other studies. Sagristà-Sauleda et al. reported only one case of CP among 461 patients with a large pericardial effusion [4]. C. Dib et al. reported 33 patients with $\mathrm{CP}$ in a large cohort of 10 years [2], Yater et al. found 3 cases among 100 non-traumatic patients with chylothorax [5]. The latest one in Beijing, China which analyzed 65 years of 92 English and Chinese studies reported 104 patients with CP in the literature [6].

$\mathrm{CP}$ occurs in all ages more dominantly in children and young adults [1]. The mean age in C. Dib study was about $36.78 \pm 14.71$ years with no gender specificity [ 1 , 2] and $27.95 \pm 16.50$ years in Yu et al. study with 5 cases younger than 3 years [6].

The disease can be diagnosed in asymptomatic patients referred for annual health examination showing a cardiomegaly or detected by dyspnea, chest pain or cough [1]. Cardiac tamponade is rare.

Most common etiologies are congenital mediastinal lymphangiectasia, iatrogenic after cardiac surgery, malignant tumors especially mediastinal ones, Gorham syndrome (defined by osteolysis resulting from lymphangiomatosis with adjacent bone resorption), blunt or penetrating trauma, infection or radiation or congenital lymphatic anomalies. Primary or idiopathic $\mathrm{CP}$ is known as a less common cause $[1,7,8]$. Idiopathic $\mathrm{CP}$ is an exclusion diagnosis when no etiology is found. This has different possible explanations proposed by Dunn and reviewed by Yu: a) Function default of lymphatic valves that are connected to the thoracic duct and pericardium lymphatic vessel; b) lymphangectasia caused by elevated pressure in the thoracic duct; c) abnormal communication between the lymphatic vessel and the pericardial sac resulting in chylous reflux; $d$ ) congenital malformation [6].

The diagnostic is usually confirmed by pericardiocentesis, followed by cytology, chemistry and culture. The fluid analysis shows a whitish or milky liquid with a high triglyceride and protein content, a predominance of lymphocytes and the presence of fat 
globules by Sudan III staining (on garde ou pas). Blood cultures had universally negative results [2]. A scoring system has been proposed by Dib et al. [2] to help establishing the diagnosis of CP. It is calculated according to the following features: i) Milky yellowish appearance of fluid; ii) TG level of $>500 \mathrm{mg} / \mathrm{dL}$; iii) Total Cholesterol/TG ratio of below 1; and iv) negative results for fluid bacterial culture and lymphocytic predominance on fluid cell count. Each feature is given 1 point and a score of 2 (specificity and sensitivity of $100 \%)$ is required for the diagnosis of CP.

Several modalities of etiological diagnosis have been reported, but lymphangiography or lymphoscintigraphy are the most used tests to detect an abnormal connection between the thoracic duct and the pericardium [1]. Lymphangiography is an invasive method including direct administration of contrast agent into cannulated lymph vessels. Combining lymphangiography and CT can be useful to detect lymphatic vessels and their relationships with the pericardial cavity. In the other hand, Lymphoscintigraphy uses radionuclides as contrast agent. It can be an alternative to lymphangiography as it is a less invasive and faster procedure.

In $\mathrm{Yu}$ et al. study, 42 (40.38\%) cases underwent lymphangioscintigraphy and 24 of them had abnormal accumulation within the pericardial sac. Among 24 cases of their series who underwent lymphangiography, 18 had abnormal findings [6]. In our study, there was no abnormal communication between the thoracic duct and pericardium. Optimal treatment for primary $\mathrm{CP}$ is still not well established. The current options are either conservative therapy including total parenteral nutrition, a low-fat diet with medium-chain triglycerides or surgical approach. The conservative one is known to be successful in post-traumatic CP [9]. It can be tried, especially if the patient is reluctant to undergo surgery or has concomitant life-limiting disease but it often fails [2]. Surgical treatment can be either a pericardiectomy, to ensure complete drainage and to prevent secondary constrictive pericarditis, or creation of a pericardial window. The latter is insufficient since it has a high recurrence rate and it does not close the communication between the thoracic duct and the pericardial sac. Ligation of the thoracic duct above the level of the diaphragm remains the best choice. Video-assisted thoracoscopic surgery is also becoming more favorable by cardiothoracic surgeons, since it is less invasive and affects the pulmonary function to a lesser extent.
Furthermore, embolism of the thoracic duct is used in certain situation, and it is an alternative type of surgery, particularly for patients who refuse to be subjected or re-subjected to more invasive approaches or those who cannot tolerate anesthesia [6].

\section{CONCLUSIONS}

Although chylopericardium is an uncommon cause of pericardial effusion, clinicians should keep this diagnosis in mind in pericarditis. Pericardiocentesis is the best way to analyze the liquid nature and help to determine the etiology. Even though, many causes can be found after investigations, idiopathic or primary $\mathrm{CP}$ is still a rare condition with only around 100 cases reported worldwide to date, and even less in Morocco.

\section{Disclosures}

None.

\section{REFERENCES}

1. Hattori H, Dakeshita E, Nakazato J, Takahashi T, Wake M, Hirata $\mathrm{K}$, et al. Primary chylopericardium treated by surgery: Report of two cases. J Cardiol Cases. 2011;3(2):e106-e10. doi: 10.1016/j.jccase.2010.12.004 pmid: 30532850

2. Dib C, Tajik AJ, Park S, Kheir ME, Khandieria B, Mookadam F. Chylopericardium in adults: a literature review over the past decade (1996-2006). J Thorac Cardiovasc Surg. 2008;136(3):650-6. doi: 10.1016/j.jtcvs.2008.03.033 pmid: 18805268

3. Pitol R, Pederiva JR, Pasin F, Vitola D. Isolated chylopericardium after cardiac surgery. Arq Bras Cardiol. 2004;82(4):384-9. doi: 10.1590/s0066-782x2004000400010 pmid: 15320559

4. Sagrista-Sauleda J, Angel J, Permanyer-Miralda G, Soler-Soler J. Long-term follow-up of idiopathic chronic pericardial effusion. N Engl J Med. 1999;341(27):2054-9. doi: 10.1056/NEJM199912303412704 pmid: 10615077

5. Yater W. Non-Traumatic Chylothorax and Chylopericardium; Review and Report of a Case Due to Carcinomatous Thromboangiitis Obliterans of the Thoracic Duct and Upper Great Veins. Annals of Internal Medicine. 1935;9(5):600. doi: 10.7326/0003-4819-9-5-600

6. Yu X, Jia N, Ye S, Zhou M, Liu D. Primary chylopericardium: A case report and literature review. Exp Ther Med. 2018;15(1):419-25. doi: 10.3892/etm.2017.5383 pmid: 29375697

7. Baratella MC, Montesello M, Marinato P. Images in cardiology. Idiopathic chylopericardium. Heart. 1998;80(4):376. doi: 10.1136/hrt.80.4.376 pmid: 9875116

8. Sleilaty G, Rassi I, Alawi A, Jebara VA. Primary isolated chronic chylopericardium. Interact Cardiovasc Thorac Surg. 2002;1(2):86-7. doi: 10.1016/s1569-9293(02)00061-0 pmid: 17669968

9. Mehrotra S, Peeran NA, Bandyopadhyay A. Idiopathic chylopericardium: an unusual cause of cardiac tamponade. Tex Heart Inst J. 2006;33(2):249-52. pmid: 16878639 